\title{
Desenvolvimento vegetativo e reprodutivo em gladíolo
}

\author{
Vegetative and reproductive development in gladiolus
}

\author{
Nereu Augusto Streck ${ }^{*}$ Rogério Antonio Bellé ${ }^{I}$ Fernanda Alice Antonello Londero Backes ${ }^{\mathrm{I}}$ Luana \\ Fernandes Gabriel ${ }^{\mathrm{II}}$ Lilian Osmari Uhlmann" ${ }^{\mathrm{III}}$ Camila Coelho Becker ${ }^{\mathrm{III}}$
}

\section{RESUMO}

A caracterização das etapas ou fases do ciclo de desenvolvimento das culturas agrícolas é importante para realização de práticas de manejo e, para o gladíolo, essas etapas ainda não foram bem caracterizadas em cultivos a campo no Rio Grande do Sul. Este trabalho teve como objetivo determinar a duração das fases vegetativa e reprodutiva do ciclo de desenvolvimento e as relações entre estas com a emissão de folhas em cultivares de gladíolo. Dois experimentos foram realizados a campo, em Santa Maria, $R S$, um na primavera/ verão 2010/11, com as cultivares: 'Priscila', 'Peter Pears', 'Sunset', 'T704', 'Traderhorn', 'Rose Supreme' $e$ 'Jester' $e$ outro no outono/inverno 2011 com as cultivares 'T704', 'Traderhorn' $e$ 'Jester'. O delineamento experimental foi inteiramente casualizado com duas repetições de cinco plantas de cada cultivar. Foram determinadas as datas do espigamento e final de florescimento e o ciclo de desenvolvimento foi dividido em duas fases: vegetativa (do plantio ao espigamento) $e$ reprodutiva (do espigamento ao final do florescimento). Os resultados indicaram que a duração da fase vegetativa é a que determina a duração do ciclo total da parte aérea e que a duração da fase vegetativa é influenciada pela velocidade de emissão das folhas e pelo número final de folhas. Assim, cultivares precoces têm maior velocidade de emissão de folhas e menor número final de folhas do que cultivares tardias.

Palavras-chave: fenologia, temperatura, soma térmica, filocrono, Gladiolus x grandiflorus.

\section{ABSTRACT}

The characterization of the developmental phases of agricultural crops is important for management practices and for gladiolus in the field this characterization has not been made in Rio Grande do Sul State. The goal of this study was to determine the duration of vegetative and reproductive phases of the developmental cycle, and the relationships between them and with leaf appearance in gladiolus cultivars. Two field experiments were conducted in Santa Maria, RS, Brazil, one during the Spring/Summer 2010/11 with the cultivars 'Priscila', 'Peter Pears', 'Sunset', 'T704', 'Traderhorn', 'Rose Supreme' and 'Jester', and another during the Fall/Winter 2011 with the cultivars 'T704', 'Traderhorn' and 'Jester'. The experimental design was a completely randomized with two replications of five plants per cultivar. The date of heading and end of flowering was determined and the developmental cycle was divided into vegetative (from planting to heading) and reproductive (from planting to and of flowering). Results showed that the duration of the vegetative phase determines the duration of the developmental cycle and the duration of the vegetative phase is affected by leaf appearance rate and by final leaf number. Thus, early cultivars have greater leaf appearance rate and lower final leaf number than late cultivars.

Key words: phenology, temperature, thermal time, phyllochron, Gladiolus x grandiflorus.

\section{INTRODUÇÃO}

O gladíolo (Gladiolus x grandiflorus Hort.), ou Palma-de-Santa-Rita, família Iridaceae, é uma flor de corte que, no Brasil, tem um importante mercado no período de Finados. As cultivares atualmente cultivadas são híbridos de várias espécies, originadas da região subsahara da África, principalmente da África do Sul (PAIVA et al., 1999). É uma planta herbácea, bulbosa, de folhas alongadas, lanceoladas com cutícula cerosa e nervuras paralelinérveas. A parte comercial do gladíolo é a inflorescência, uma espiga com vários floretes, em

'Departamento de Fitotecnia, Centro de Ciências Rurais (CCR), Universidade Federal de Santa Maria (UFSM), Av. Roraima, 1000, 97105-900, Santa Maria, RS, Brasil. E-mail: nstreck1@smail.ufsm.br. *Autor para correspondência.

"IPrograma de Pós-graduação em Agronomia, UFSM, Santa Maria, RS, Brasil.

${ }^{\text {IIIC } C u r s o ~ d e ~ A g r o n o m i a, ~ U F S M, ~ S a n t a ~ M a r i a, ~ R S, ~ B r a s i l . ~}$ 
uma variedade de cores incluindo branco, amarelo, vermelho, lilás e verde. Por ser uma cultura de ciclo curto, fácil condução, baixo custo de implantação e rápido retorno financeiro, é recomendada para cultivo em pequenas áreas, com vistas ao mercado interno e exportação (PAIVA et al., 1999). No Brasil, o cultivo em grande escala do gladíolo iniciou na década de 50 , incentivado pela Cooperativa Agrícola Holambra. Em 1989, o cultivo já era notório em São Paulo, com primeiro lugar em consumo interno, terceiro em flores cultivadas e quarto em exportação (TOMBOLATO et al., 2004).

Crescimento e desenvolvimento vegetal são processos distintos que, ao longo da estação de cultivo, podem ocorrer simultaneamente ou não. Crescimento refere-se ao aumento de dimensões físicas de partes ou órgãos da planta, como, por exemplo, a área de folhas, o comprimento de caule e o volume de frutos, enquanto desenvolvimento refere-se a eventos envolvendo diferenciação celular, iniciação de órgãos (organogênese), aparecimento de órgãos (morfogênese) e se estende até a senescência da cultura, como, por exemplo, a diferenciação floral, o aparecimento de folhas e a antese (HODGES, 1991; STRECK et al., 2003). Geralmente, o ciclo de desenvolvimento das culturas agrícolas pode ser dividido em duas fases: vegetativa e reprodutiva (McMASTER, 2005; STRECK et al., 2006). Essa divisão é baseada em indicadores morfológicos facilmente identificáveis a campo, a fase vegetativa inicia na emergência das plantas e acaba quando a estrutura reprodutiva fica visível ao observador a olho nu, quando começa a fase reprodutiva, que se estende até a senescência da cultura (STRECK et al., 2006). Durante a fase vegetativa, ocorre o aparecimento de folhas (ou desenvolvimento foliar), que está diretamente relacionado com a área foliar do dossel, a qual intercepta radiação solar para fotossíntese, e com a evapotranspiração da cultura. Em gramíneas, a duração da fase vegetativa e do ciclo total é proporcional ao número final de folhas na haste principal, enquanto que a duração da fase reprodutiva dessas culturas não tem relação com a duração da fase vegetativa, indicando que o melhoramento vegetal tem concentrado esforço em caracteres que mantém a duração da fase reprodutiva, independente do grupo de maturação (precoce ou tardia) (STRECK et al., 2006).

A caracterização das etapas ou fases do ciclo de desenvolvimento das culturas agrícolas é importante, pois práticas de manejo como adubação de cobertura, tratamentos fitossanitários, época e lâmina de irrigação e planejamento da colheita têm maior eficiência quando baseadas no momento em que as plantas se encontram no ciclo de desenvolvimento do que quando baseadas em dias do calendário civil (ex. dias após o plantio, dias após a emergência, dias após a poda). Por exemplo, na cultura do arroz, as duas adubações de nitrogênio em cobertura são recomendadas em V4 (quatro folhas) e na diferenciação da panícula (SOSBAI, 2010). Em culturas florículas, essa ideia de realizar práticas culturais com base no ciclo de desenvolvimento é igualmente aplicada, bem como no planejamento de épocas de plantio para escalonar a produção e reduzir a sazonalidade da oferta de flores no mercado. Mas, para isso, deve-se conhecer e entender detalhadamente as diferentes etapas do ciclo de desenvolvimento de cada cultura. As etapas do ciclo de desenvolvimento da parte aérea do gladíolo ainda não foram bem caracterizadas em cultivos a campo no Rio Grande do Sul, o que constitui a motivação para esta pesquisa. $\mathrm{O}$ objetivo deste trabalho foi determinar a duração da fase vegetativa e da fase reprodutiva do ciclo de desenvolvimento e as relações entre estas com a emissão de folhas em cultivares de gladíolo.

\section{MATERIAL E MÉTODOS}

Dois experimentos de campo foram realizados na área experimental do Setor de Floricultura do Departamento de Fitotecnia da Universidade Federal de Santa Maria durante a primavera/verão de 2010/11 e o outono/inverno de 2011. No primeiro experimento, sete cultivares de gladíolo foram avaliadas: 'Priscila' (ciclo precoce, cor rosa), 'Peter Pears' (ciclo médio, laranja), 'Sunset' (ciclo médio, amarela), 'T704' (ciclo médio, lilás), 'Traderhorn' (ciclo médio, vermelha), 'Rose Supreme' (ciclo tardio, salmão), 'Jester’ (ciclo tardio, amarela com interior vermelho). O plantio dos cormos (com circunferência equatorial entre 12 e $14 \mathrm{~cm}$ para as cultivares 'Rose Supreme' e 'T704', e entre 14 e 16cm para as cultivares 'Traderhorn', 'Jester', 'Peter Pears', 'Sunset' e 'Priscila') ocorreu no dia 08/09/2010 na profundidade de $8 \mathrm{~cm}$. O espaçamento foi de $20 \mathrm{~cm}$ entre plantas e $30 \mathrm{~cm}$ entre linhas pareadas em um canteiro de $1 \mathrm{~m}$ de largura. No segundo experimento, foram avaliadas três cultivares ('T704', 'Traderhorn' e 'Jester') e o plantio foi em 30/03/2011 no espaçamento de $20 \mathrm{~cm}$ entre plantas e $40 \mathrm{~cm}$ entre linhas. Os bulbos variaram de 5 a $12 \mathrm{~cm}$, na cultivar 'T704', 8 a 20cm na cultivar 'Traderhorn' e, na cultivar 'Jester', os cormos tinham $12 \mathrm{~cm}$.

O delineamento experimental nos dois experimentos foi inteiramente casualizado com duas repetições, sendo cada parcela constituída de cinco plantas. A adubação seguiu as recomendações da cultura (TOMBOLATO et al., 2004). As plantas daninhas foram controladas através de capinas e o solo foi irrigado para evitar o estresse hídrico. Em todas as plantas da parcela, foram determinadas as datas do espigamento (EP: espiga visível no cartucho de folhas) e final do florescimento (FF: último florete aberto e com as anteras visíveis na extremidade apical da espiga). $\mathrm{O}$ 
ciclo de desenvolvimento foi dividido em duas fases: vegetativa (do Plantio-PL até o EP) e reprodutiva (do EP até o FF). Durante a fase vegetativa, foi contado semanalmente o número de folhas acumuladas (NFA) e, no final do experimento, o número final de folhas (NFF) na haste de cada planta. A soma térmica diária (STd, ${ }^{\circ} \mathrm{C}$ dia) a partir da emergência foi calculada por (ARNOLD, 1960): STd=[(Tmax + Tmin)/2]-Tb, em que Tmax e Tmin são as temperaturas máxima e mínima diária do $\operatorname{ar}\left({ }^{\circ} \mathrm{C}\right)$, medidas na Estação Climatológica Principal da UFSM, localizada a aproximadamente 200m da área experimental, e Tb é a temperatura base da cultura, considerada $7^{\circ} \mathrm{C}$ (VIDALIE, 1990). A soma térmica acumulada (STa, ${ }^{\circ} \mathrm{C}$ dia) foi calculada por: $\mathrm{STa}=\Sigma \mathrm{STd}$.

$\mathrm{O}$ filocrono (intervalo de tempo entre o aparecimento de duas folhas sucessivas, um indicador da velocidade de emissão de folhas) $\left[{ }^{\circ} \mathrm{C}\right.$ dia folha $\left.{ }^{-1}\right]$ em cada planta foi calculado pelo inverso do coeficiente angular da regressão linear entre o NFA e a STa (XUE et al., 2004; STRECK et al., 2009). Foi calculada também a duração, em ${ }^{\circ} \mathrm{C}$ dia, das fases vegetativa (PL-EP) e reprodutiva (EP-FF). A análise estatística constou da análise de variância e teste Tukey, para as variáveis filocrono e NFF, e de análise de regressão linear simples entre duração ( ${ }^{\circ} \mathrm{C}$ dia) das fases vegetativa e reprodutiva versus ciclo total (PL-EF) e entre duração da fase vegetativa versus filocrono e NFF, conforme STRECK et al. (2006), ao nível de significância de 5\%. Para as variáveis filocrono e NFF, foram considerados dois experimentos. Na análise de regressão, usou-se o primeiro experimento para estimar o coeficiente linear e o angular da regressão e o segundo experimento como dados independentes para testar a regressão estimada no primeiro experimento e verificar se as relações entre as fases do ciclo de desenvolvimento, filocrono e NFF determinados no cultivo de primavera/verão se mantêm no cultivo de outono/inverno, isto é, a robustez das relações na interação genótipo x ambiente.

\section{RESULTADOS E DISCUSSÃO}

No primeiro experimento, a data do EP variou de 19/11/2010 na cultivar 'Priscila' a 05/12/2010 na cultivar 'Jester' e a data do FF variou de 10/12/2010 na cultivar 'Priscila' até 28/12/2011 na cultivar 'Rose Supreme'. No segundo experimento, a data do EP variou de 29/06/2011 na cultivar 'Traderhorn' a 18/07/2011 na cultivar 'Jester' e a data do FF variou de 08/08/2011 na cultivar ‘Traderhorn' a 25/08/2011 na cultivar 'Jester'. A temperatura mínima diária do ar variou de $5,3^{\circ} \mathrm{C}$ a $22,8^{\circ} \mathrm{C}$ e de $-0,8^{\circ} \mathrm{C}$ a $20,1^{\circ} \mathrm{C}$ e a temperatura máxima diária do ar variou de $16,4^{\circ} \mathrm{C}$ a $37^{\circ} \mathrm{C}$ e de $9,6^{\circ} \mathrm{C}$ a $34,8^{\circ} \mathrm{C}$ no primeiro e segundo experimentos, respectivamente. As diferentes cultivares e épocas de plantio proporcionaram exposição das plantas a condições distintas de ambiente, o que é importante em estudos de fenologia e para testar a robustez das relações entre duração das diferentes fases do ciclo de desenvolvimento.

No primeiro experimento, o filocrono variou de 87,4 a $130,8^{\circ} \mathrm{C} \mathrm{dia}^{-1}$ folha $^{-1}$ nas cultivares 'Peter Pears' (que não diferiu das cultivares 'Priscila', 'Sunset', 'Rose Supreme’ e 'Jester’) e ‘T704' (que não diferiu da cultivar 'Traderhorn'), respectivamente (Tabela 1). O NFF variou de 7,7 a 9,8 folhas nas cultivares ‘Traderhorn' (que não

Tabela 1 - Filocrono $\left({ }^{\circ} \mathrm{C}\right.$ dia folha ${ }^{-1}$ ) e número final de folhas (NFF) na haste principal de sete cultivares de gladíolo no primeiro experimento (primavera/verão de 2010/11) e de três cultivares no segundo experimento (outono/inverno de 2011). Santa Maria, RS, 2010.

\begin{tabular}{|c|c|c|}
\hline Cultivar & Filocrono & NFF \\
\hline \multicolumn{3}{|c|}{ 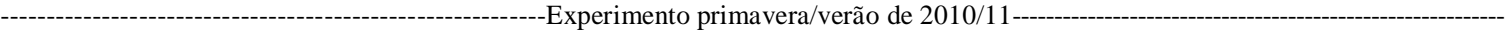 } \\
\hline 'Priscila’ & $89,5 c^{*}$ & $8,0 \mathrm{ab}$ \\
\hline 'Peter Pears’ & $87,4 \mathrm{c}$ & 9,8 a \\
\hline 'Sunset’ & 94,0 bc & 9,5 a \\
\hline ‘T704’ & 130,8 a & $9,0 \mathrm{ab}$ \\
\hline 'Traderhorn' & $112,6 \mathrm{ab}$ & 7,7 b \\
\hline 'Rose Supreme’ & 96,3 bc & $9,3 \mathrm{ab}$ \\
\hline ‘Jester’ & 106,5 bc & 9,7 a \\
\hline CV (\%) & 10,8 & 11,7 \\
\hline 'T704' & $84,1 \mathrm{~b}$ & 8,4 a \\
\hline ‘Traderhorn’ & $69,9 \mathrm{~b}$ & 9,2 a \\
\hline ‘Jester’ & $100,5 \mathrm{a}$ & 8,8 a \\
\hline CV (\%) & 13,1 & 6,6 \\
\hline
\end{tabular}

*Médias seguidas pela mesma letra minúscula na vertical em cada experimento não diferem pelo teste Tukey a 5\% de erro. CV = coeficiente de variação. 
diferiu das cultivares 'Priscila', 'T704' e 'Rose Supreme') e 'Peter Pears' (que diferiu apenas de 'Traderhorn'), respectivamente (Tabela 1). A fase vegetativa teve duração variando de 849,5 a $1112,7^{\circ} \mathrm{C}$ dia nas cultivares 'Priscila' e 'Jester', respectivamente, enquanto a duração da fase reprodutiva variou de 215,7 a $264,0^{\circ} \mathrm{C}$ dia nas cultivares 'Jester' e 'Sunset', respectivamente. $\mathrm{O}$ ciclo total (PL-FF) expresso em dias teve a sequência 'Priscila' < 'Peter Pears' < 'T704' < 'Traderhorn' < 'Sunset' < 'Jester' < 'Rose Supreme' (Figura 1A) e, quando expresso em ${ }^{\circ} \mathrm{C}$ dia, a sequência foi 'Priscila' < 'Peter Pears' < 'Sunset' < 'Traderhorn' < 'T704' < 'Jester' < 'Rose Supreme' (Figura 1B). No segundo experimento, o filocrono da cultivar 'Jester' diferiu das cultivares 'Traderhorn' e 'T704' e o NFF não diferiu entre as três cultivares (Tabela 1 ). A duração da fase vegetativa variou de 922,9 a $1041,5^{\circ} \mathrm{C}$ dia e a duração da fase reprodutiva variou de 287,3 a $312,5^{\circ} \mathrm{C}$ dia nas cultivares 'Traderhorn' e 'Jester', respectivamente (Figura 1B). A duração do ciclo total em ${ }^{\circ} \mathrm{C}$ dia teve a sequência 'Traderhorn' < 'T704' < 'Jester' e, em dias, a sequência foi 'T704' < 'Traderhorn' < 'Jester' (Figura 1A).

A duração, em dias, do ciclo total do segundo experimento teve um aumento considerável comparado ao primeiro experimento (Figura 1A), no qual a fase vegetativa aumentou de 3,8 e 22 dias e a fase reprodutiva aumentou de 21,16 , e 15 dias nas cultivares 'T704', 'Traderhorn' e 'Jester', respectivamente. A duração mais longa da fase reprodutiva indica vantagem de comercialização da inflorescência nos meses mais frios nas regiões subtropicais. A redução da fase vegetativa ( $\mathrm{em}^{\circ} \mathrm{C}$ dia) no experimento outono/ inverno pode ser atribuída ao fotoperíodo mais curto neste período do ano, comparado com o período primavera/ verão, pois o gladíolo é considerado uma planta de dia

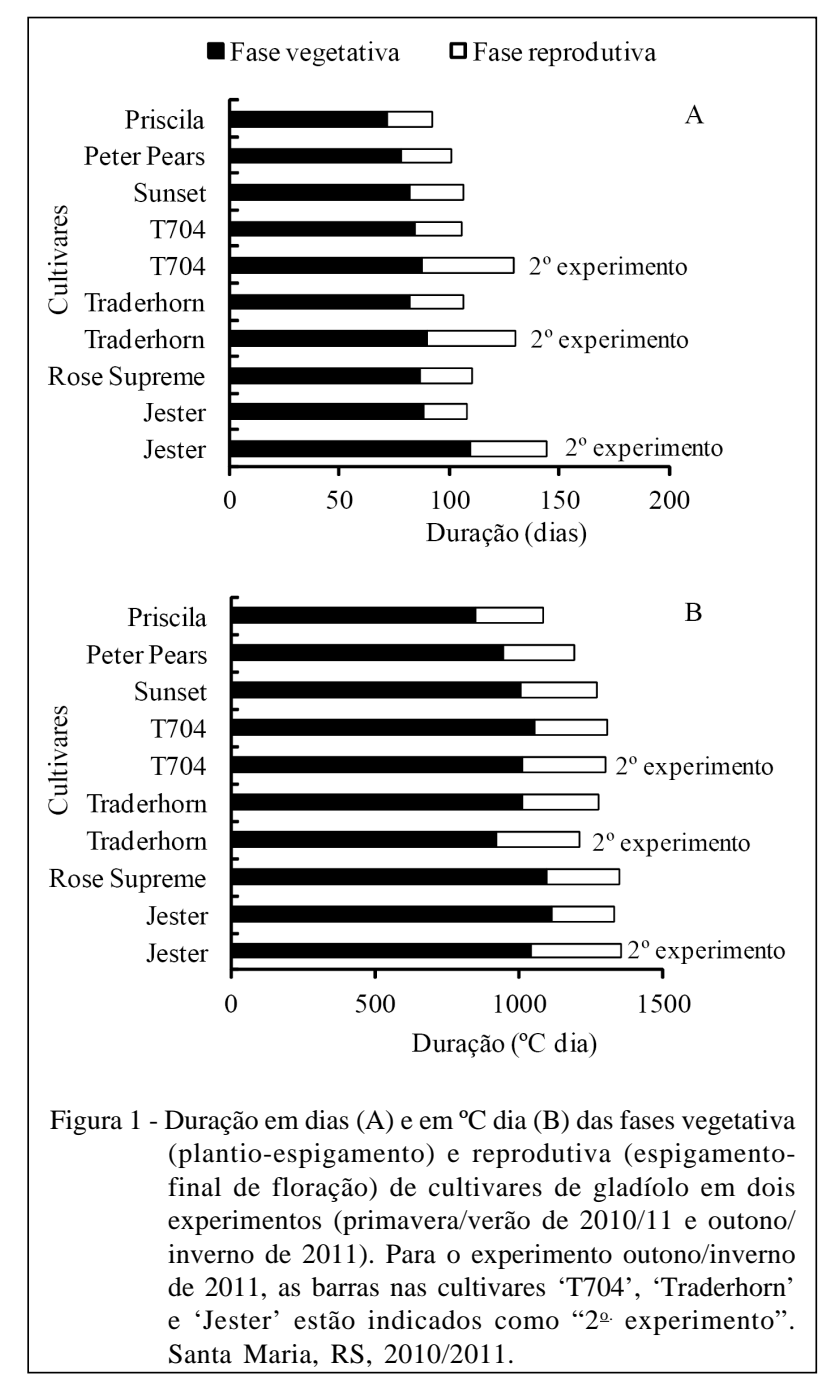

Ciência Rural, v.42, n.11, nov, 2012. 
curto facultativa (SHILLO \& HALEVY, 1976). A variação entre duração do ciclo em dias e em ${ }^{\circ} \mathrm{C}$ dia entre os experimentos está relacionada com a temperatura, pois, em temperaturas mais baixas, o ciclo de desenvolvimento das culturas se alonga (caso do segundo experimento, outono/inverno), enquanto dias com temperaturas mais altas encurtam o ciclo (caso do primeiro experimento, primavera/verão) (STRECK et al., 2003). Por isso, a caracterização do ciclo em ${ }^{\circ} \mathrm{C}$ dia é mais apropriada, pois esta unidade de tempo leva em consideração a condição térmica do dia (STRECK et al., 2009).

No primeiro experimento, a duração do ciclo total (PL-FF) teve uma relação linear positiva significativa, com $\mathrm{R}^{2}=0,89$, com a duração da fase vegetativa (PL-EP) (Figura 2A), enquanto que não houve relação entre a duração do ciclo total com a duração da fase reprodutiva (EP-FF) (Figura 2B). Esses resultados indicam que a duração do ciclo total da parte aérea do gladíolo é uma função direta e positiva da duração da fase vegetativa, ou seja, quanto maior a fase vegetativa, maior a duração do ciclo total. A duração da fase vegetativa, por sua vez, teve uma relação com a velocidade de emissão de folhas, representada pelo filocrono $\left(\mathrm{R}^{2}=0,30 ; \mathrm{P}<0,0009\right.$ ) (Figura $2 \mathrm{C}$ ) e com o NFF $\left(R^{2}=0,36 ; P<0,0002\right)$ (Figura 2D), ou seja, quanto maior o filocrono (menor velocidade de emissão de folhas) e quanto maior o NFF, mais longa é a fase vegetativa. Esses resultados concordam com os resultados encontrados por STRECK et al. (2006) em arroz e por ZANON et al. (2012) em trigo, espécies monocotiledôneas, nas quais o ciclo total é definido (afetado) pela duração da fase vegetativa e pelo NFF no colmo principal, mas em trigo (ZANON et al., 2012) e em arroz (STRECK et al., 2006) a duração da fase vegetativa não teve relação com o filocrono, diferindo do gladíolo. $\mathrm{O}$ baixo valor de $\mathrm{R}^{2}$ nas figuras $2 \mathrm{C}$ e $2 \mathrm{D}$, comparados com $o \mathrm{R}^{2}$ na figura $2 \mathrm{~A}$, estão associados à menor influência da velocidade de aparecimento de folhas sobre a

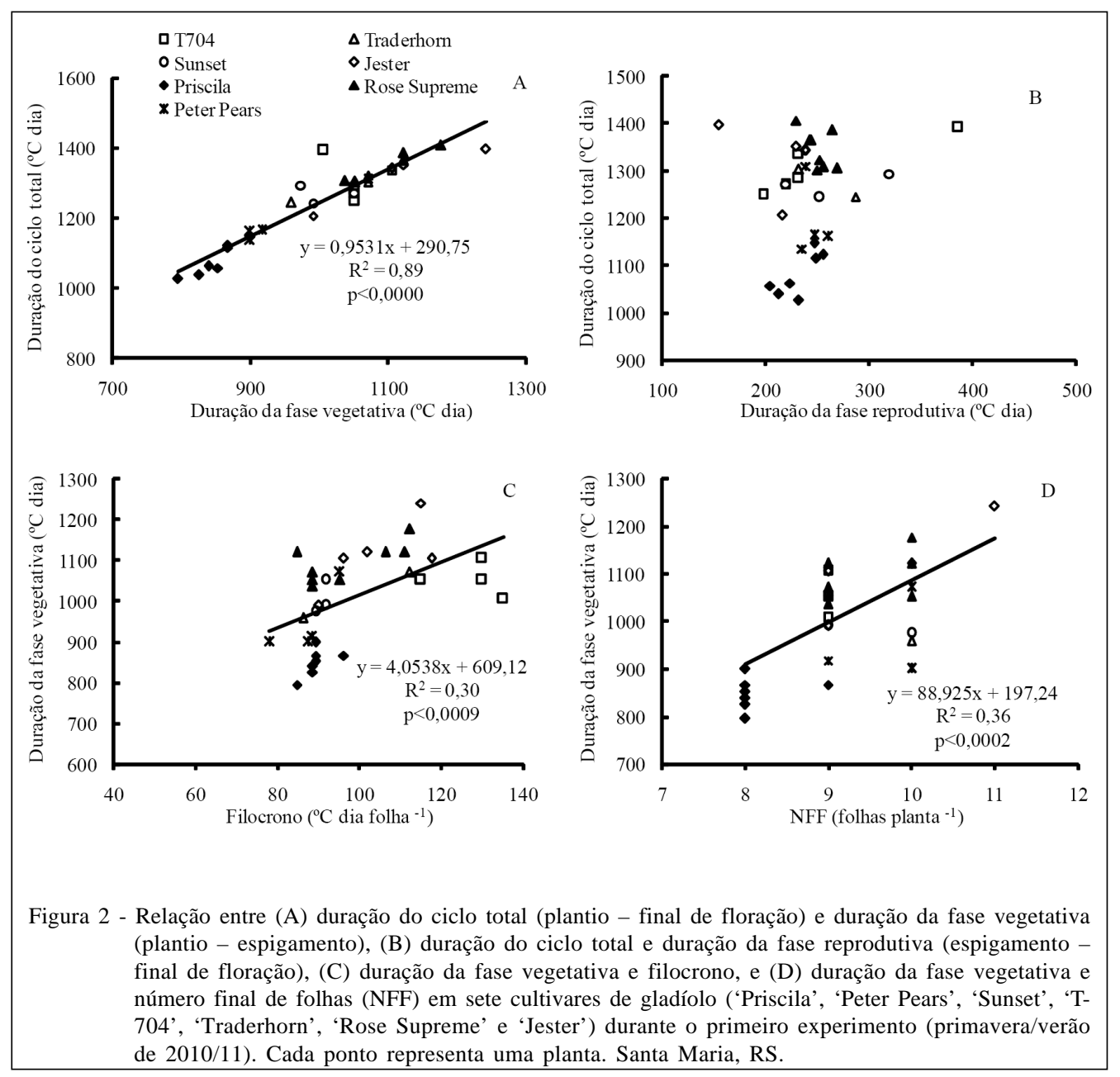

Ciência Rural, v.42, n.11, nov, 2012. 
duração da fase vegetativa, o que também foi constatado em arroz (STRECK et al., 2009) e em trigo (ZANON et al., 2012), e devido ao fato do NFF ser uma variável discreta (foi considerada por planta e não a média da parcela).

Na figura 3, estão plotadas as relações entre duração das fases vegetativa e reprodutiva versus ciclo total (Figura 3A e 3B) e filocrono e NFF versus fase vegetativa (Figura 3C e 3D) do segundo experimento (outono/inverno de 2011), com a linha das regressões ajustadas aos dados deste experimento (Figura 3A e 3B) e com a linha das regressões lineares estimadas do primeiro experimento (Figura 2A, 2C e 2D). Observa-se que os pontos se situam próximo das linhas de tendência do primeiro experimento, principalmente para a relação fase vegetativa versus ciclo total (Figura 3A) e fase vegetativa versus filocrono (Figura 3C), mas também para fase vegetativa versus NFF (Figura 3D) e que a relação ciclo total versus fase reprodutiva é inexistente (Figura 3B), sendo os coeficientes angulares na figura 3A e 3C próximos nos dois experimentos, confirmando as relações (ou falta de relação) do primeiro experimento. Destaca-se a relação entre duração da fase vegetativa (Figura 3C) em que há um considerável número de pontos com filocrono menor que $80^{\circ} \mathrm{C}$ dia folha ${ }^{-1}$ fora da faixa em que a linha de tendência foi estimada no primeiro experimento, o que indica, do ponto de vista de modelo matemático, alta capacidade preditiva e, do ponto de vista fisiológico, alta dependência da velocidade de emissão das folhas, com a duração da fase vegetativa até o surgimento da espiga floral e, portanto, alta robustez das relações estimadas na figura 2. Para a figura $3 \mathrm{D}$, a regressão não foi significativa no segundo experimento, devido ao pequeno número de cultivares e apenas três plantas com NFF=10 da cultivar 'Traderhorn', que, no primeiro experimento, também apresentou NFF=10 folhas. Isso não significa que a relação obtida no primeiro

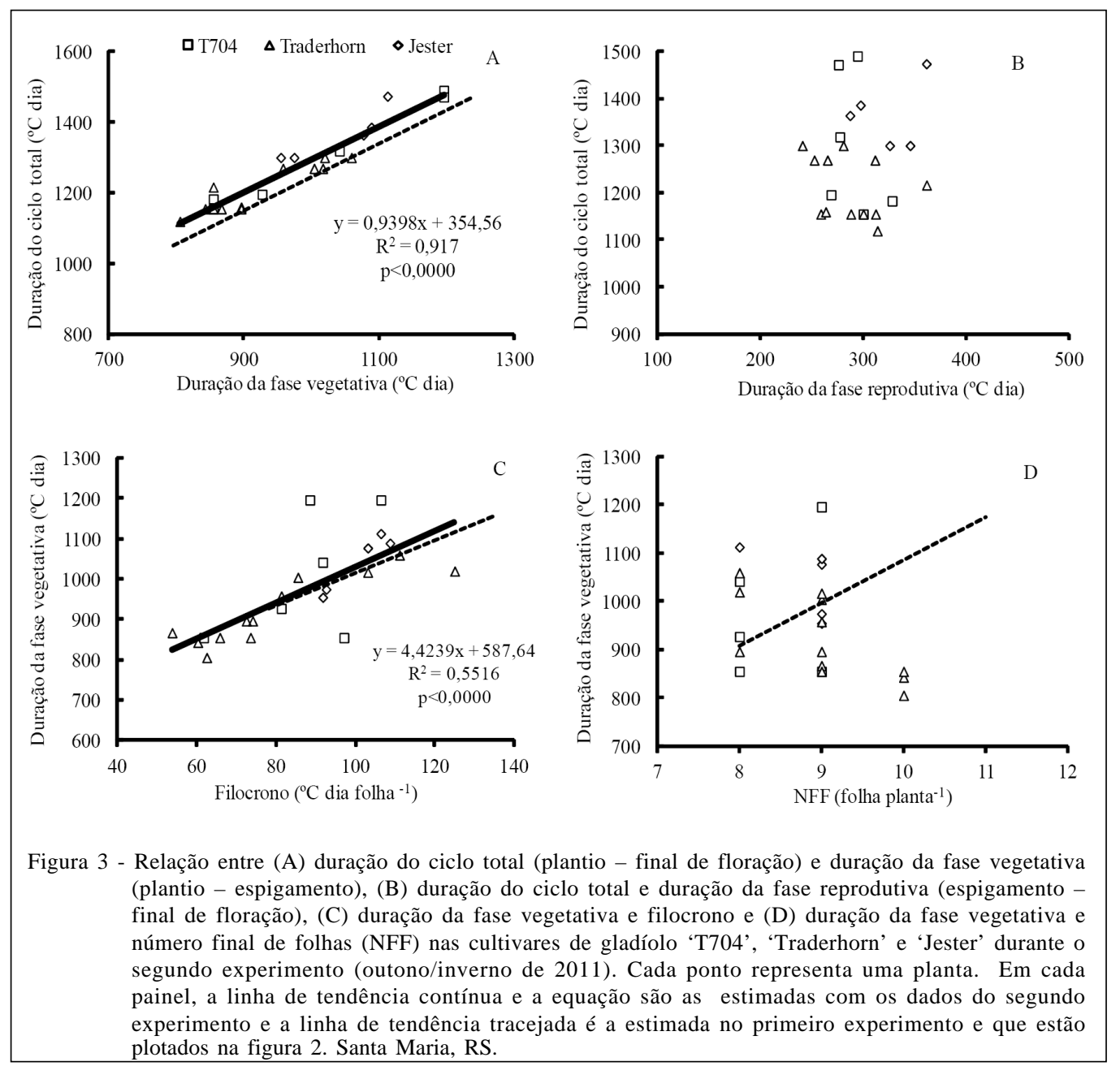

Ciência Rural, v.42, n.11, nov, 2012. 
experimento não se manteve no segundo experimento, pelo contrário, para NFF de 8 e 9 a relação obtida no primeiro experimento (Figura 2D) se repetiu na figura 3D. A implicação desses resultados em programas de melhoramento de gladíolo é que a seleção de genótipos para precocidade de floração pode ser feita com base nos caracteres velocidade de emissão de folhas e número final de folhas, facilmente medidos a campo.

\section{CONCLUSÃO}

A duração da fase vegetativa de gladíolo até o espigamento tem relação linear positiva com a duração do ciclo total e, morfologicamente, é determinada pela velocidade de emissão e número final de folhas, enquanto que a duração da fase reprodutiva (EP-FF) não tem relação com o ciclo total. Cultivares precoces têm maior velocidade de emissão de folhas (menor filocrono) e menor número final de folhas do que cultivares tardias.

\section{REFERÊNCIAS}

ARNOLD, C.Y. Maximum-minimum temperatures as a basis for computing heat units. Proceedings of the Americam Society for Horticultural Sciences, v.76, n.1, p.682-692, 1960.

HODGES, T. Predicting crop phenology. Boca Raton: CRC, 1991. 233p.

McMASTER, G.S. Phytomers, phyllochrons, phenology and temperate cereal development. Journal of Agricultural Science, v.143, p.137-150, 2005. Disponível em: <http:// dx.doi.org/10.1017/S0021859605005083>. Acesso em: 19 jan. 2012. doi: 10.1017/S0021859605005083.

PAIVA, P.D.O. et al. Cultura do gladíolo. Lavras: UFLA Departamento de Agricultura, 1999. 12p.

SHILLO, R.; HALEVY, A.H. The effect of various environmental factors on flowering of gladiolus. II-Length of the day. Scientia
Horticulturae, v.4, p.139-146, 1976. Disponível em: <http:/ /dx.doi.org/10.1016/S0304-4238(76)80005-2>. Acesso em: 20 jan. 2012. doi: 10.1016/S0304-4238(76)80005-2.

SOSBAI. Arroz irrigado: recomendações técnicas da pesquisa para o Sul do Brasil/28. Reunião técnica da cultura do arroz irrigado. Porto Alegre, 2010. 188p.

STRECK, N.A. et al. Improving predictions of developmental stages in winter wheat: a modified Wand and Engel model. Agricultural and Forest Meteorology, v.115, p.139-150, 2003. Disponível em: <http://dx.doi.org/10.1016/S01681923(02)00228-9>. Acesso em: 05 jan. 2012. doi: 10.1016/ S0168-1923(02)00228-9.

STRECK, N.A. et al. Duração do ciclo de desenvolvimento de cultivares de arroz em função da emissão de folhas na coluna principal. Ciência Rural, v.36, n.4, p.1086-1093, 2006. Disponível em: <http://www.scielo.br/pdf/cr/v36n4/a07v36n4.pdf>. Acesso em: 12 jan. 2012. doi: 10.1590/S0103-84782006000400007.

STRECK, N.A. et al. Temperatura base para aparecimento de folhas e filocrono da variedade de milho BRS Missões. Ciência Rural, v.39, n.1, p.224-227, 2009. Disponível em: <http:// dx.doi.org/10.1590/S0103-84782009000100035>. Acesso em: 02 jan. 2012. doi: 10.1590/S0103-84782009000100035.

TOMBOLATO, A.F.C. et al. Gladíolo. In: TOMBOLATO, A.F.C. Cultivo comercial de plantas ornamentais. Campinas: Instituto Agronômico, 2004. Cap.5, p.137-173.

VIDALIE, H. Les productions florales. 6.ed. Paris: Lavoisier, 1990. 249p.

XUE, Q. et al. Predicting leaf appearance in field-grown winter wheat: evaluating linear and non-linear models. Ecological Modelling, v.175, p.261-270, 2004. Disponivel em: <http:/ /dx.doi.org/10.1016/j.ecolmodel.2003.10.018>. Acesso em: 12 jan. 2012. doi: 10.1016/j.ecolmodel.2003.10.018.

ZANON, A.J. et al. Número de folhas associado com duplo anel e espigueta terminal em cultivares de trigo. Revista Ciência Agronômica, v.43, p.569-578, 2012. Disponível em: <http:/ /www.ccarevista.ufc.br/seer/index.php/ccarevista/article/ viewFile/1607/720>. Acesso em: 16 maio, 2012. 